
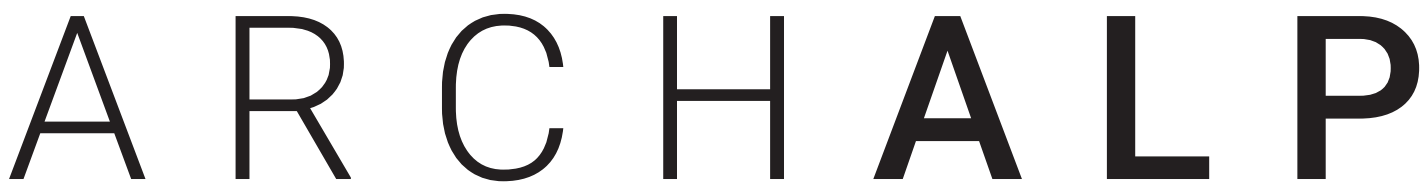

Rivista internazionale di architettura e paesaggio alpino / Revue internationale d'architecture et de paysage dans les Alpes / Internationale Zeitschrift für Alpine Architektur und Landschaft / Revija za alpsko arhitekturo in pokrajino / International journal of alpine architecture and landscape

Rer una nuota abitabilita delle Alpi.

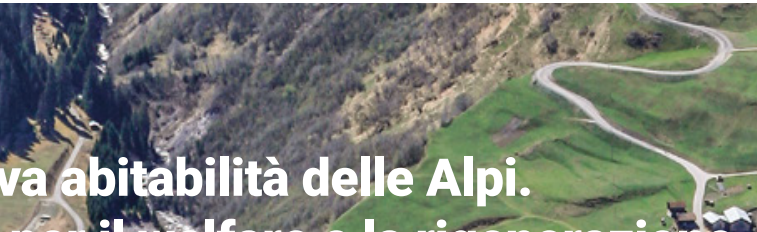
Architeture pet ilf lfare e la rigenerazione For a new inhabifability of the Alps. Architectures for welfare ând regeneration $/$ Pour une nouvelle habitabilité des Alpes. Architectures pour le welfare et la régéhérationy Für eine núue Bewohnbarkeit in den Alpen. Architekturen für Wohf ahrt und Regeneration / Za novo. bivalnost v Alpah, arhitekture za dobrobit in regeneracijo
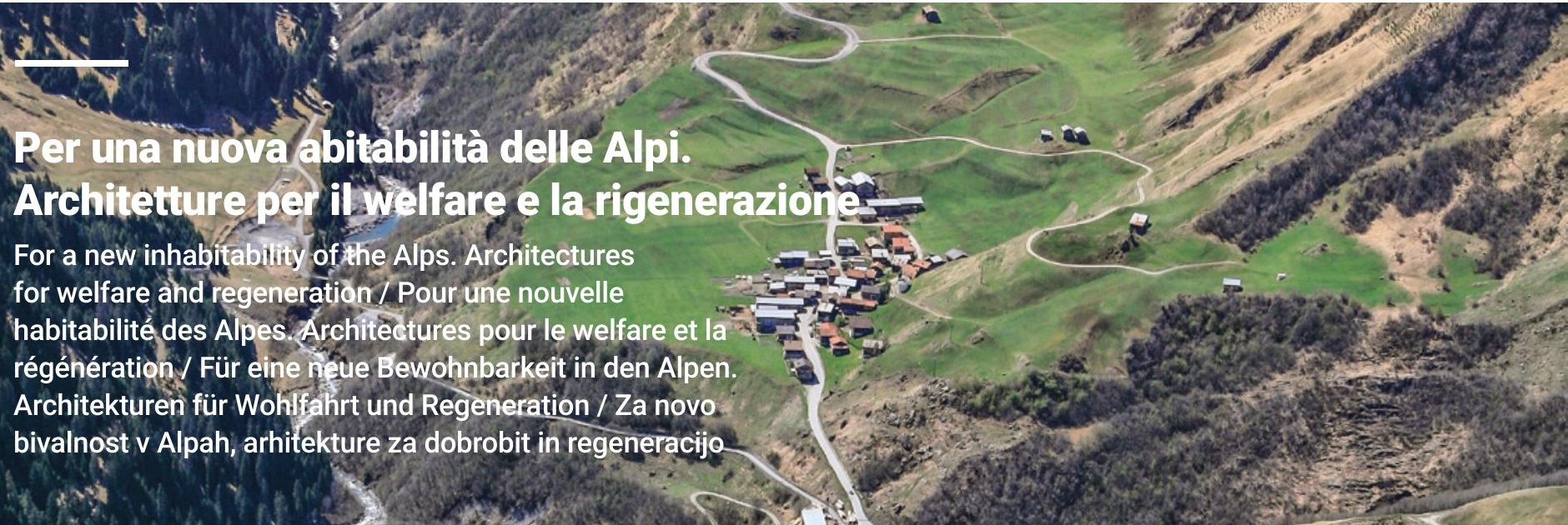
a $x \rightarrow 3$
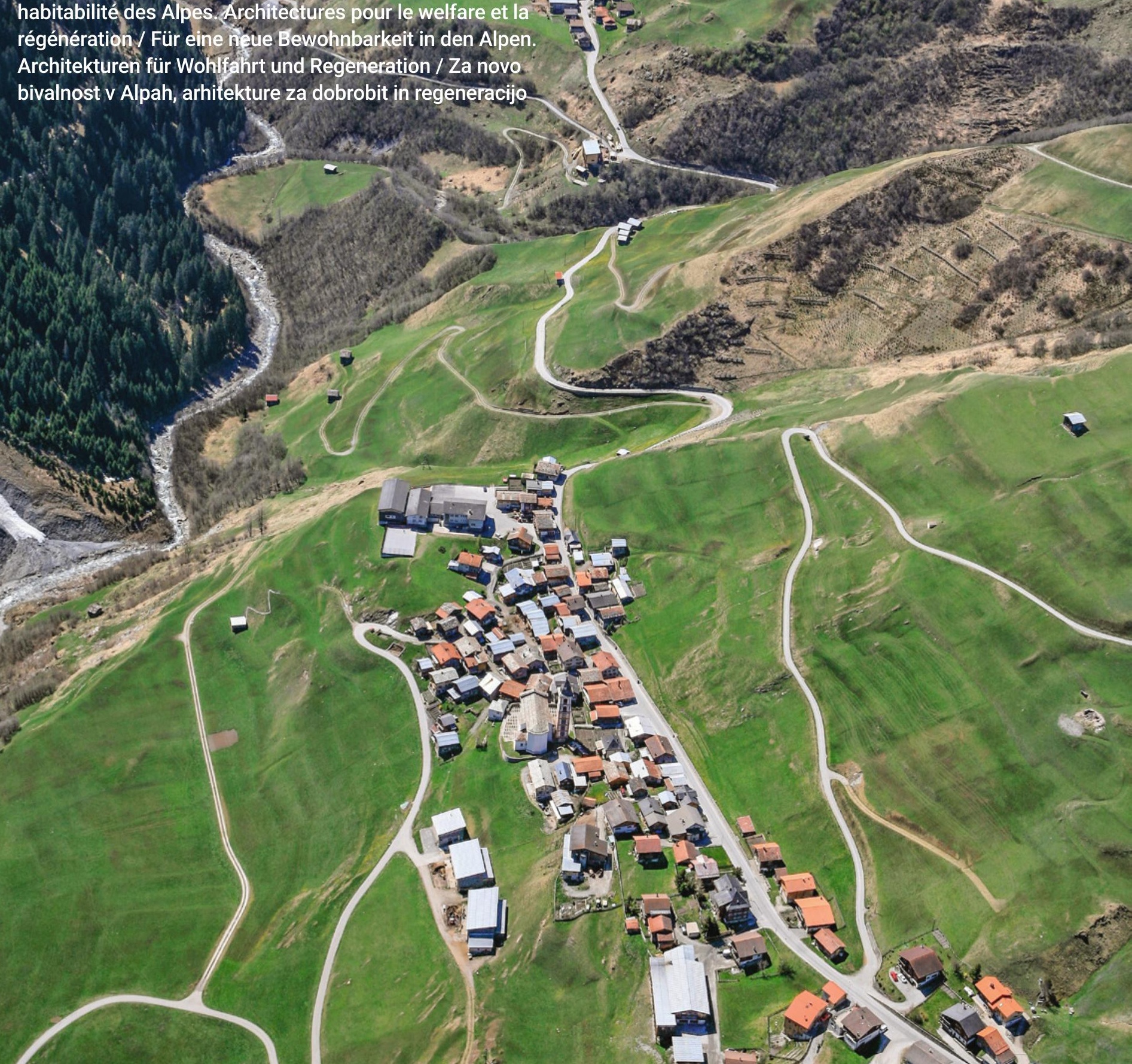


\section{Indice dei contenuti Contents}

\section{Temi}

Sulla centralità di spazio e territorio nel progetto di rigenerazione delle montagne e delle aree interne / On the centrality of space and territory in the project of regeneration of mountains and internal areas Antonio De Rossi, Laura Mascino

I servizi nelle Alpi italiane: quali e dove? Idee per uno scenario post-pandemico / Facilities in the Italian Alps: which ones and where? Ideas for a post-pandemic scenario

Giuseppe Dematteis

Manifesto di Camaldoli per una nuova centralità della montagna

Alla ricerca della distanza perduta. Rigenerare luoghi, persone e immaginari del riabitare alpino / In search of the lost distance. Regenerating places, people and images related to Alpine reinhabitation

Filippo Barbera, Andrea Membretti

Futuro e rigenerazione

Marco Bussone

\section{Esperienze}

Dorferneuerung zwischen Erhalten und Gestalten / 
Valades ousitanes, architettura e rigenerazione / Valades ousitanes,

Ostana e Topolò: hardware, software e welfare nelle comunità di "ritorno" / Ostana and Topolò: hardware, software and welfare in "return" communities

Margherita Valcanover

"Senza mostrare i muscoli": i progetti di rigenerazione di Tao+C e AZL nel solco della "prosperosa società" della Cina contemporanea / "Without flexing one's muscles": the regeneration projects of Tao+C and AZL in the wake of the "prosperous society" of contemporary China Edoardo Bruno, Dalila Tondo

L'archipel Butor. Une régénération, par la culture, d'un village soumis à la métropolisation genevoise / The Butor archipelago. A regeneration, through culture, of a village subject to the metropolisation of Geneva Arnaud Dutheil

Pratiche e progettualità di rigenerazione e welfare: il "Premio triennale Giulio Andreolli - Fare paesaggio" / Actions and projects of regeneration and welfare: the "Premio triennale Giulio Andreolli - Fare paesaggio" Giorgio Tecilla

Architetture e strategie per il welfare. Il caso di Brunico in Val Pusteria / Welfare architectures and strategies. The Bruneck case in Val Pusteria

Eleonora Gabbarini

Arhitektura oživlja / Architecture revives Kristina Dešman, Maja Ivanič

Si Crans-Montana meurt. Soigner le corps malade d'une station / If Crans-Montana dies. Taking care of the ailing body of a tourist resort Patrick Giromini 


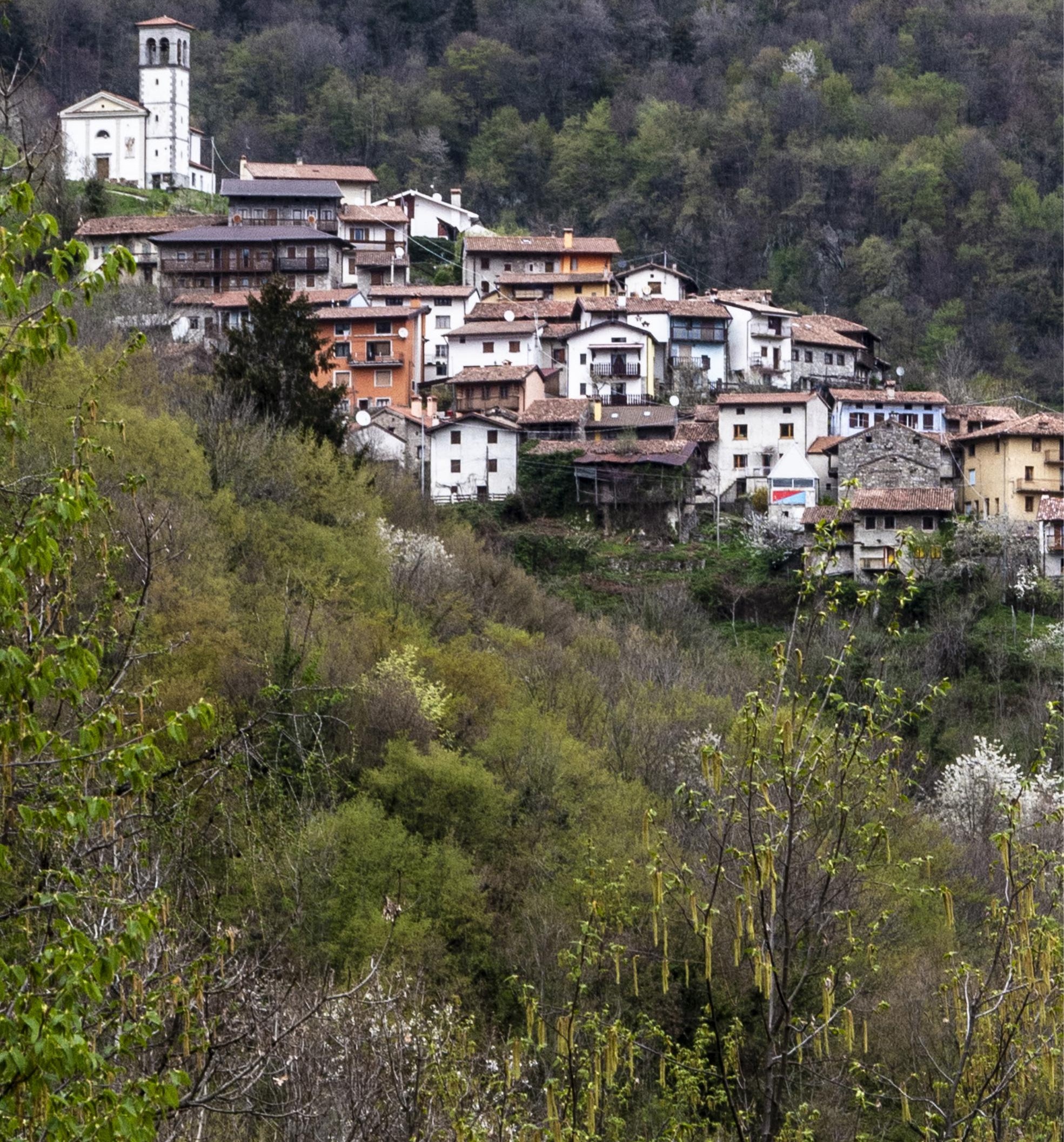




\title{
Ostana e Topolò: hardware, software e welfare nelle comunità di "ritorno"
}

\author{
Ostana and Topolò: hardware, software and welfare in "return" \\ communities
}

\begin{abstract}
Ostana and Topolò are two small villages at the far ends of the Alps that have a macroscopic point in common, not so obvious for Highland areas: the reversal of the trend of demographic decline. It is not just about numbers, but rather meanings: for these communities to be reborn, it was crucial to act simultaneously both on the tangible dimension of the place - with the restoration and reuse of the architectural heritage - and on the intangible one, with the introduction of new activities, functions and collective usage. The widespread design and restoration of the architectural heritage, together with the presence of aggregative and social-spatial foci, is what characterizes these two experiences. These spaces, located within the settlement fabric, are configured as reference points for the community: they are an expression of a need still present in the contemporary world, and are themselves, in turn, capable of generating new processes. Casa Juliova in Topolò and Lou Pourtoun in Ostana are the most significant social foci of the two villages: two different ways of interpreting the reuse of the local architectural heritage, both objects of a "memory and creation strategy" (Choay, 1995). The two experiences demonstrate how the "physical" intertwining between culture and welfare is a winning strategy in the revitalization and self-centered regeneration of Highland areas. Are marginal territories ready to face the next socio-environmental challenges?
\end{abstract}

\section{Margherita Valcanover}

Architect, graduated from the IUAV University of Venice, and currently a PhD student at the Politecnico di Torino, where she researches the forms of architectural reuse that characterize the return to the mountains.
Keywords

Vernacular architecture, heritage, reuse, social regeneration, Alpine communities, Alpine welfare. 
1. I ritornanti, grazie alle loro intuizioni, sperimentazioni e innovazioni, danno vita ad esperienze anche molto interessanti dell'abitare la montagna contemporanea. Ciò nonostante, la maggior parte delle volte rimangono, per scelta, per forza o per sfortuna, concluse nella loro singolarità. La qualità architettonica con cui attuano operazioni di riuso adattivo del patrimonio, per lo più ma non solo, afferente all'ambito del mondo rurale, è spesso raffinata e di qualità. A oggi si incomincia a vedere una certa casistica di pratiche di rigenerazione, ma non tutte sfociano poi nella formazione di una comunità, almeno nel senso tradizionale del termine, attiva. Quali casi sono stati invece capaci di formare una comunità? Cosa vuol dire welfare nelle comunità più periferiche? Qual è stato il ruolo del progetto di architettura e dello spazio? Con quali modalità specifiche si è attuato il riuso del patrimonio?

Questo articolo parla di Ostana e Topolò, due casi paradigmatici di come sia possibile arrivare a costituire una comunità in evoluzione, ma tutto sommato stabile, a partire dalla costruzione di un hardware architettonico e un software culturale. Grazie infatti ad un duplice lavoro sul patrimonio architettonico e sulla programmazione di attività artistiche, culturali e turistiche ad ampio respiro, è stato possibile creare attorno a questi centri un fermento sociale che poi ha portato alla formazione di una comunità coesa, partecipativa e fortemente radicata. Sia per le operazioni materiali che per quelle immateriali, la radice metodologica si trova nella conoscenza della storia, soprattutto locale, da cui, con «fantasia e pazienza» (Varotto, 2013) è germinata la costruzione della realtà odierna. Gli interventi puntuali sul patrimonio per un uso collettivo, o sociale, sono arrivati solo dopo che il processo di "stabilizzazione" della comunità ha raggiunto un buon livello di maturazione. Per quanto riguarda questi ultimi, sono stati individuati dei fuochi all'interno del tessuto edilizio da adibire appunto a spazi di uso sociale. A Ostana troviamo ad esempio il Lou Pourtoun e la nuova casa alpina Mizoun de la Villo, a Topolò la Postaja, l'ambasciata di Olanda e gli stessi vuoti interstiziali. Welfare in queste comunità sono soprattutto dei luoghi "necessari" in cui la comunità abbia la possibilità e la libertà di lavorare in maniera sia individuale che collettiva. In questi luoghi si tengono incontri, seminari, piccoli convegni, corsi di vario tipo, si suona, si fanno le prove per recite, si accolgono i visitatori, si espongono opere, ecc. Casa Fuliova e Lou Pourtoun non sono solo singoli oggetti, ma entrano piuttosto in una sequenza di spazi e in un sistema di relazioni. In questo network, si può dire che essi siano gerarchicamente i fuochi più importanti, dove converge l'orientamento, ma senza il loro contesto materiale e immateriale sarebbero oggetti fluttuanti nel nulla. Alla scala architettonica, lo studio dell'evoluzione morfologica delle strutture insediative, assieme

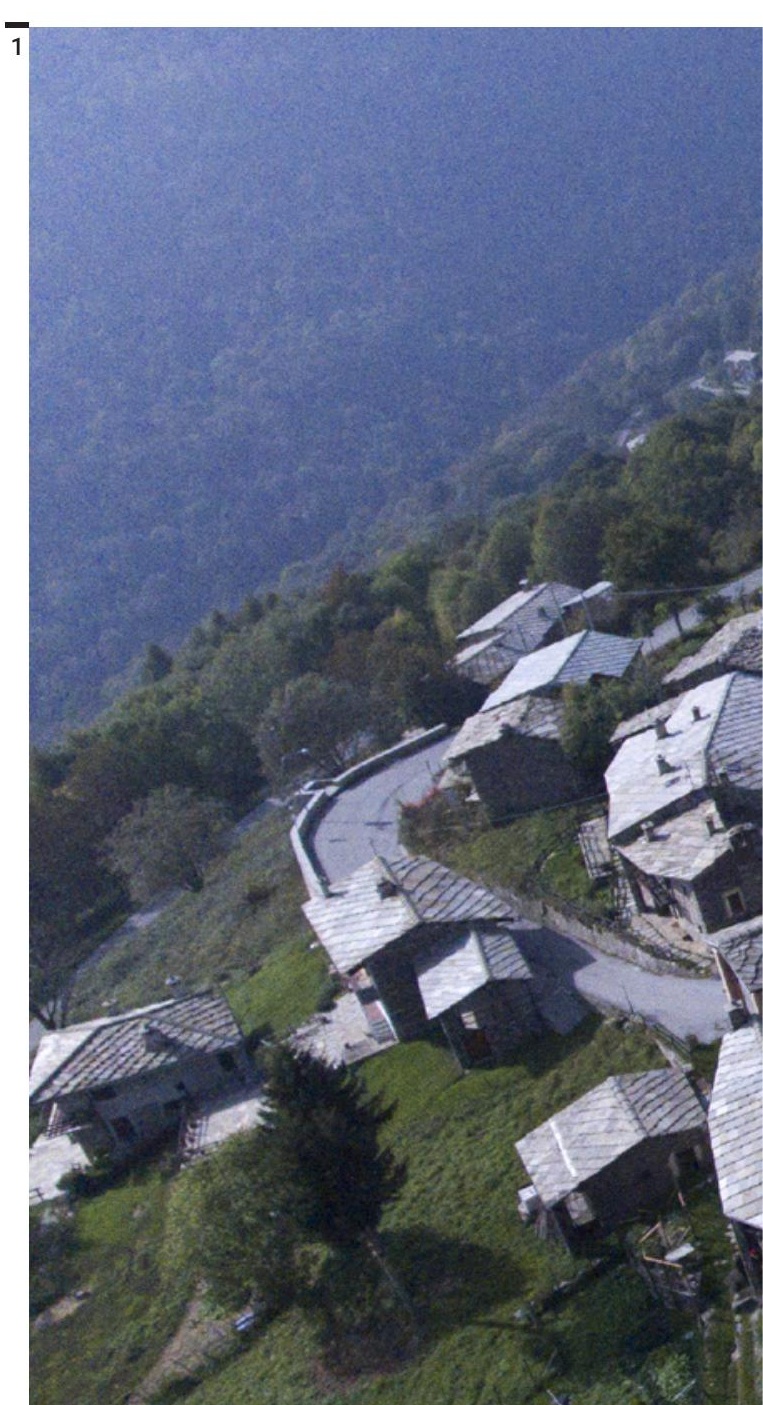


alla conoscenza materiale degli oggetti fisici, sono gli elementi in comune che hanno condotto a degli originali e significativi processi di traduzione, finanche di invenzione, del patrimonio e di conseguente rigenerazione. In Abbecedario del restauro, il riuso viene definito come «la parte essenziale di un programma progettuale che implica la capacità di comprendere la storia, significato, condizioni e vocazioni dell'esistente per proporre un ruolo rinnovato all'interno del contesto urbano e territoriale» (Dezzi Bardeschi, 2017). In entrambi i casi, lo studio e la conoscenza storica preliminare hanno portato ad esplicare due modalità di intervento sul patrimonio apparentemente simili, ma che celano una prassi architettonica anche molto differente una dall'altra, soprattutto sull'interpretazione delle architetture vernacolari e quindi nella loro rimessa in efficienza.

Casa fuliova a Topolò storicamente era la casa privata e padronale di merciai ambulati che distribui-

Fig. 1

Vista della frazione di S. Antonio,

Ostana, Cuneo

(foto Margherita Valcanover)
Ora casa Juliova, sede della Pinacoteca universale di To- $^{-}$ polò, è un luogo dove si ospitano i visitatori durante il festival, si svolgono riunioni, attività musicali, corali, si espongono opere d'arte ecc. Tutte le strade conducono alla Pinacoteca si potrebbe dire parafrasando, tanto è fondamentale la sua presenza nel tessuto urbano del paese. Per quanto riguarda gli esterni, l'arch. Rucli ha mantenuto il paramento murario pietra a vista sui lati sud, est e ovest, mentre al lato nord ha riproposto una peculiarità delle architetture di Topolò: l'intonaco colorato sulla strada pubblica. Sul lato sud ed est ha ripristinato il ballatoio ligneo. Per quanto riguarda l'interno sono stati mantenuti dei particolari originali come la Pec (stufa), i decori sulle travi e i pavimenti. La distribuzione spaziale tra i piani e i settori della casa è stata liberata da quella originale, un artista visuale olandese ha aggiunto delle decorazioni murarie concettuali ispirate al luogo. Gli esterni della fuliova sono stati progettati anch'essi per diventare a sud, sotto il ballatoio ligneo, uno spazio espositivo, a nord una sorta di cavea con palchetti composta dai gradoni di accesso all'edificio.

Il Lou Pourtoun a Ostana invece è per certi versi un'architettura inesistente. Non è un riuso in senso stret-

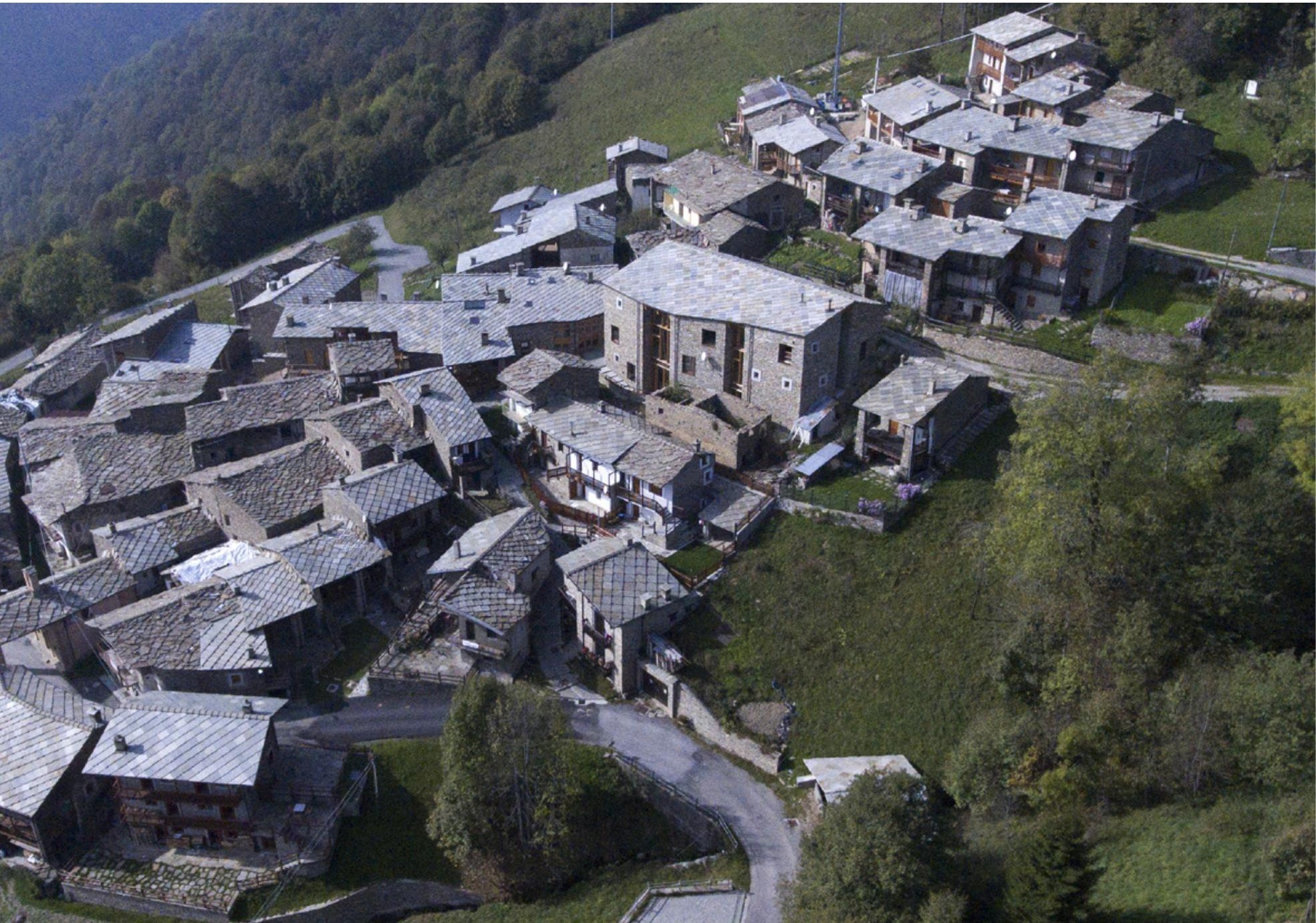



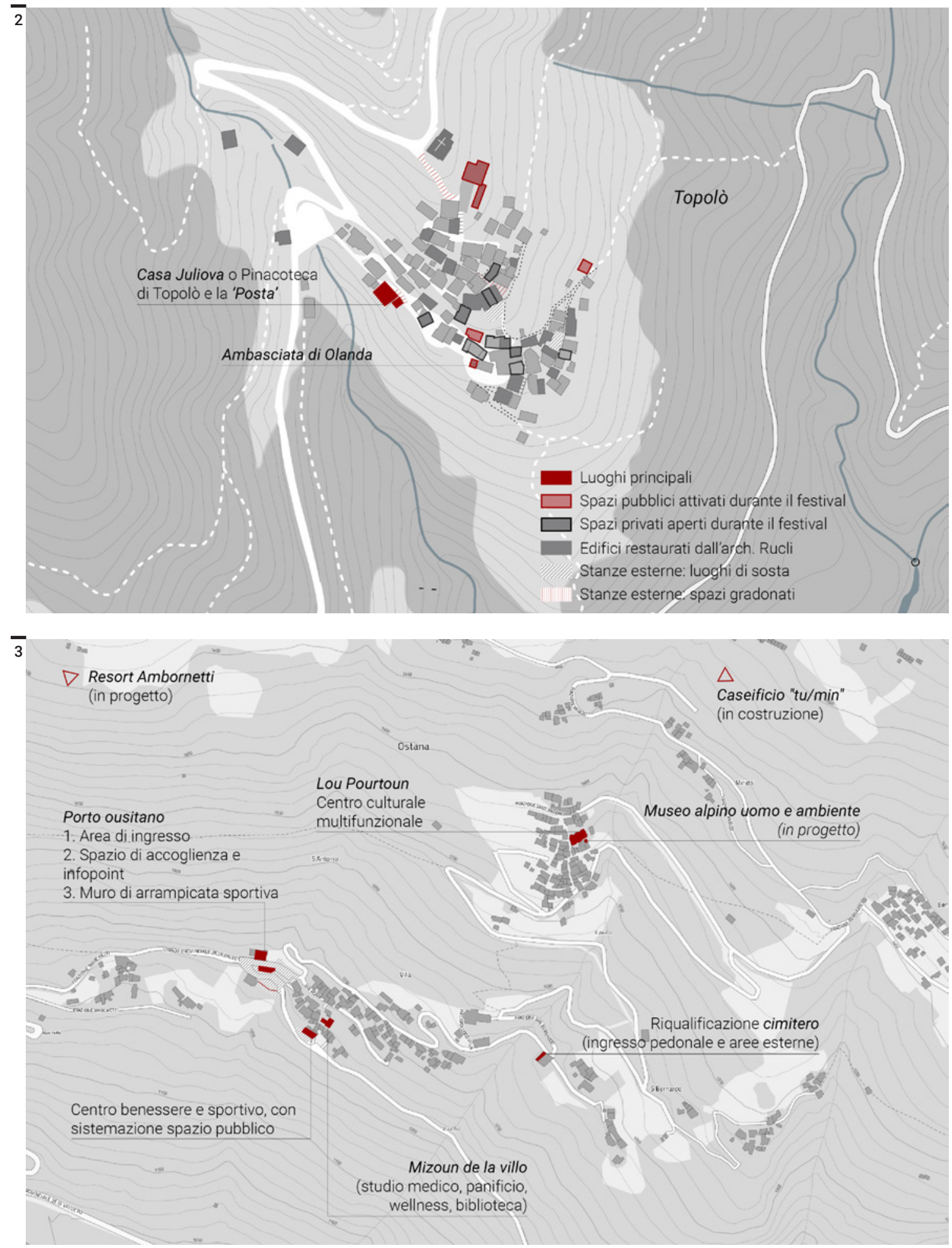

Fig. 3

Planimetria di

Ostana con gli interventi di rigenerazione realizzati o avviati a partire dal 2008

(elaborazione grafica Margherita Valcanover). to, in quanto le preesistenze erano crollate, ma la riproposizione e l'interpretazione dello studio storico-morfologico del sistema distributivo dell'ex mercato coperto del paese. Il riuso è quindi, da un punto di vista progettuale, una mera ricostruzione planimetrica di una preesistenza. Laddove vi erano i pieni, è stata riproposta una struttura muraria, mentre dove vi erano i vuoti è stata riconfigurata la distribuzione interna, tamponata con ampie finestre a tutta parete. $\mathrm{Al}$ piano terra e al primo piano vi è una ritmica alternanza di spazi flessibili che possono essere utilizzati come aule e sale riunioni, al piano interrato vi è una sala polifunzionale molto ampia. La copertura, in lose di pietra locale, e il riuso del pietra- 


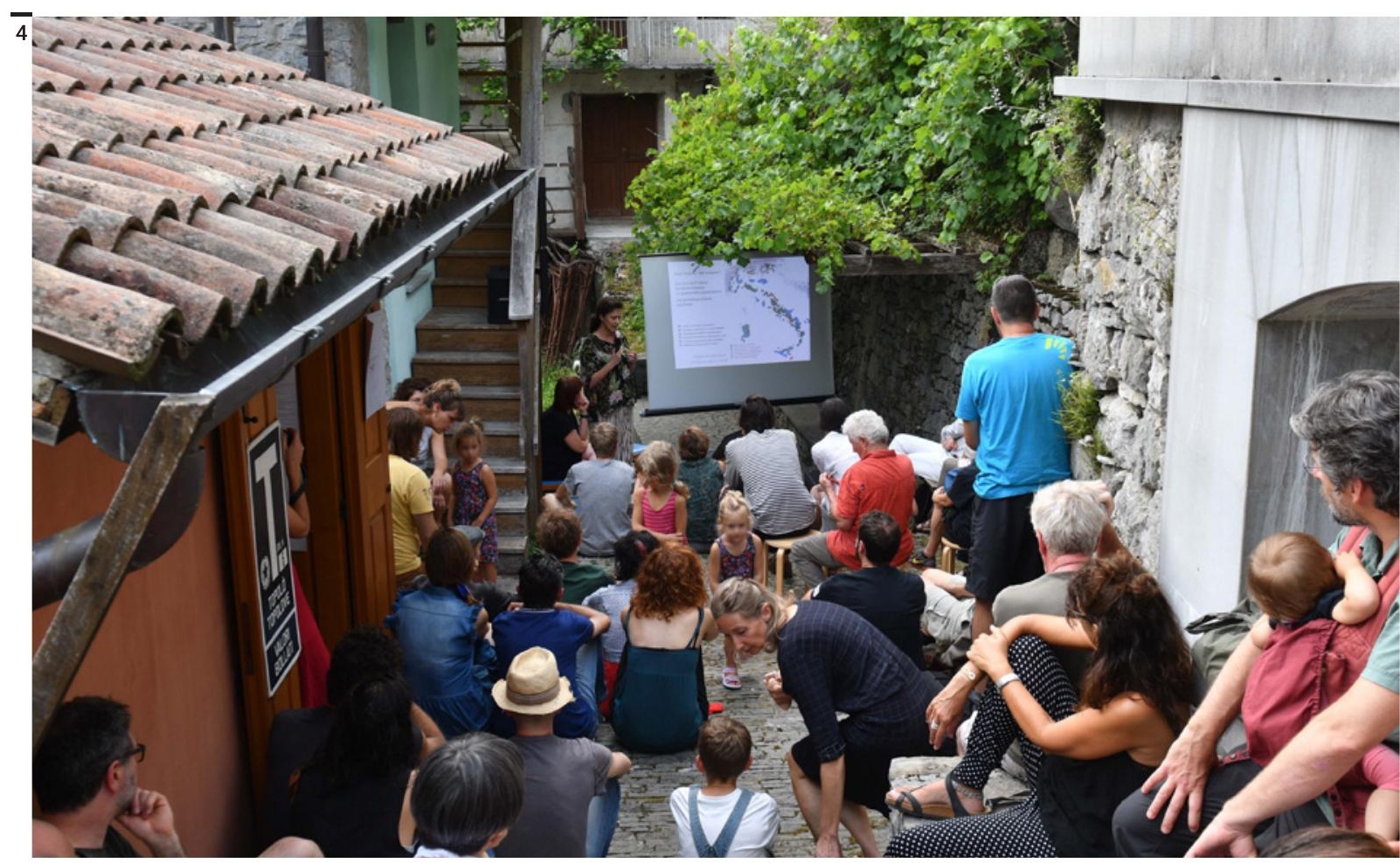

me delle preesistenti murature a secco conferiscono all'edificio una finta mimesi, svelata dall'introduzione appunto delle grandi vetrate e dalle altre aperture che la proiettano così tanto nell'architettura contemporanea da vincere numerosi premi di architettura, tra cui il prestigioso Constructive Alps nel 2017.

2. Ostana è il comune più piccolo della Valle Po in provincia di Cuneo, Piemonte. Come quasi tutti i comuni alpini, nel corso del Novecento, ha subito anch'esso forti dinamiche di spopolamento a favore dei centri più grossi di fondo valle. Tendenza che all'inizio degli anni Novanta ha cominciato a cambiare rotta: dai 6 abitanti rimasti nel momento più basso della curva demografica agli 80 circa di oggi. Dalla fine degli anni Ottanta le varie amministrazioni hanno messo in atto progettualità di lunga durata che vedevano in tre capisaldi lo sviluppo del proprio territorio: la promozione della cultura occitana, un nuovo modello di turismo e il recupero e la valorizzazione del patrimonio architettonico e paesaggistico (Crotti, 2016). Sempre a partire dagli anni Ottanta si sono attuati, in più fasi, vari interventi di recupero dell'architettura locale destinati prevalentemente ad abitazioni private. Interventi caratteriz-

Fig. 4

Poste e Casa Juliova. Utilizzo degli spazi esterni durante il Festival di Topolò

(foto Margherita

Valcanover). zione. Negli ultimi dieci anni, nel momento in cui la comunità di Ostana compiva i primi passi di stabilizzazione, sono state costruite diverse strutture a destinazione pubblica, affrontando quindi un programma di intervento più ampio rispetto alle sole ristrutturazioni a scopo residenziale. Esse sono state possibili grazi a programmi di finanziamento europei, nazionali e regionali per lo sviluppo locale.

3. Forse un po' meno conosciuta, almeno da un punto di vista bibliografico, ma altrettanto interessante, è la storia di Topolò-Topolove. Il paese è una piccola frazione del comune di Grimacco nelle Valli del Natisone, in Friuli-Venezia Giulia, al confine tra l'Italia e la Slovenia. Adagiato su un ripido crinale a circa $600 \mathrm{~m}$ slm si inserisce in un contesto complesso, e dal punto di vista geografico e orografico, e dal punto di vista storico-culturale: qui il Novecento non è stato un secolo breve, aleggiano ancora gli echi di tutte le guerre del secolo scorso, con gli spostamenti dei confini e di ciò che ne consegue. In sincronia con tutte le Aree interne, anche qui arriva il dramma dello spopolamento, acuito anche da eventi catastrofici come i terremoti, che tanto hanno martoriato le terre friulane nel secolo scorso.

E così, in un clima sociale difficile, a metà degli anni Novanta, un vivace trio, composto da due architetti e un curatore d'arte, iniziò ad interrogarsi su cosa si sarebbe potuto fare per portare un'azione pacificante ad un trauma di cui essenzialmente si era persa la natura, per dirla con Zanzotto. Uno di questi ar- 
chitetti, Renzo Rucli, aveva già compiuto uno studio storico-morfologico dell'architettura vernacolare locale, definendone con precisione i caratteri specifici (Gariup, 1994). La risposta che trovarono fu nell'esperienza artistica inventando il festival Stazione To- $^{-}$ polò-Postaja Topolove, e chiamarono solo quegli artisti che potevano dialogare in maniera attenta, umile e silenziosa col paesaggio e con la storia locale. Le situazioni espositive si collocano all'interno degli edifici, spesso in abbandono, nei loro spazi interstiziali, e un po' più lontano dentro al paesaggio che circonda il paese. Tutti spazi progettati e riadattati ad hoc, per essere funzionali ad un utilizzo collettivo.

Anno dopo anno il festival, la cui durata è di circa due settimane, si ripete e si rinnova, allarga la sua rete, si fissano nella trama del paese i fuochi di riferimento, luoghi come la Pinacoteca, la Posta, l'ambasciata di Svezia, che vengono restaurati e messi a disposizione del pubblico. Contemporaneamente si sedimenta nelle persone un senso di attaccamento così radicato e radicale che fa sì che più persone prendano a cuore quest'iniziativa. Alcuni decidono di credere a questo progetto investendo su primi edifici ricettivi seguendo nel restauro le indicazioni dell'arch. Rucli e che altri, giovani specialmente, eleggono a residenza proprio quel luogo apparentemente lontano dall'urbanesimo contemporaneo. Vi è stata l'esperienza anche di un'occupazione di una

Fig. 5

Topolò, restauro del patrimonio architettonico privato, architetto Renzo Rucli

(foto Margherita Valcanover).

Fig. 6

Ostana, Lou Pourtoun. Architetti Massimo Crotti, Antonio De Rossi, MariePierre Forsans (foto Margherita Valcanover). fessionalità, ma soprattutto quanto sia importante la sopravvivenza di paesaggi innanzitutto colmi di senso. Per far sì che ciò avvenga è auspicabile che torni nelle prassi di sviluppo strategico dei territori la progettazione fisica dello spazio come un'operazione culturale a stretto contatto con la storia locale e con le necessità della contemporaneità, soprattutto quelle espresse dalle forze in campo. Il progetto culturale in senso esteso è l'elemento chiave che tiene insieme le pratiche, i manufatti ed i servizi. E l'appropriazione del patrimonio culturale è l'esito di una volontà collettiva. «Oggi [...] possiamo riconoscere la definizione concreta del welfare non può che essere l'esito instabile di un processo interattivo che si autoalimenta» (Secchi, 2011).

Per quanto gli esiti non possano essere modellizzabili, proprio a causa dell'instabilità di ciascuna situazione, il metodo e il processo progettuale analizzati segna un punto fisso di buona pratica per uno sviluppo autocentrato del territorio. In un possibile scenario futuro di ritorno massivo alla montagna bisognerà capire esattamente cosa e come consegnare al futuro per far sì che la montagna possa generare sempre significato, e quindi tramandarsi. La montagna non è solo un grande serbatoio di servizi ecosistemici, ma è anche un serbatoio di manufatti il cui valore viene "reiventato" volta per volta, "The real care of the Alpine heritage therefore lies in its permanent reinvention» (Jakob, 2019). Questa possibilità di rigenerazione è l'unica maniera per far sì che il valore patrimoniale e sociale dell'architettura e del paesaggio alpino non si svaluti mai nel corso delle epoche e delle mode. Ci vuole tempo per costruire un progetto di territorio e di comunità, e l'architettura si inserisce quando questo processo di costruzione è stabile e maturo, e più vi è l'immersione nella storia locale più la qualità si alza. Così, è successo così nel Voralberg, in val Lumnezia, in Bregaglia. Cambiano gli esiti architettonici, ma il metodo è all'incirca lo stesso: il punto di partenza è la comunità, le sue esigenze e i luoghi dove esplicarle, ma per partire dalla comunità bisogna avere una visione progettuale e politica del territorio, e quindi di strategie di sviluppo.

\section{Bibliografia}

Choay Françoise (1995), L'allegoria Del Patrimonio, Officina Edizioni, Roma.

Clavuot Conradin (2016), I/ rapporto con la storia nell'architettura alpina, in Del Curto Davide, Dini Roberto, Menini Giacomo, Alpi. Architettura. Patrimonio, progetto, sviluppo locale, Mimesis, Milano.

Crotti Massimo (2016), Valorizzare i borghi alpini: il caso di Ostana in Valle Po, in Del Curto Davide, Dini Roberto, Menini Giacomo, Alpi. Architettura. Patrimonio, progetto, sviluppo locale, Mimesis, Milano.

De Rossi Antonio (2019), Ostana: architettura e rigenerazione. https://www.wearch.eu/ostana-architettura-e-rigenerazione/ (maggio 2020).

Dezzi Bardeschi Chiara (2017), Riuso, in Ead. (a cura di), Abbecedario Minimo'Ananke. Cento Voci per II Restauro, Altralinea Edizioni, Firenze.

Gariup Mario, Gariup Renzo, Rucli Renzo (1994), Topolò. Topolove. Racconto sulle origini di un paese delle Valli del Natisone, Circolo culturale Rean Liessa Grimacco, Cooperativa "Lipa” s.r.l., Mariano del Friuli.

Jakob Michael (2019), «ll paesaggio alpino in quanto oggetto patrimoniale», in ArchAlp, n. 2 ns - Manipolazioni metasemiche del patrimonio, pp. 35-41. 

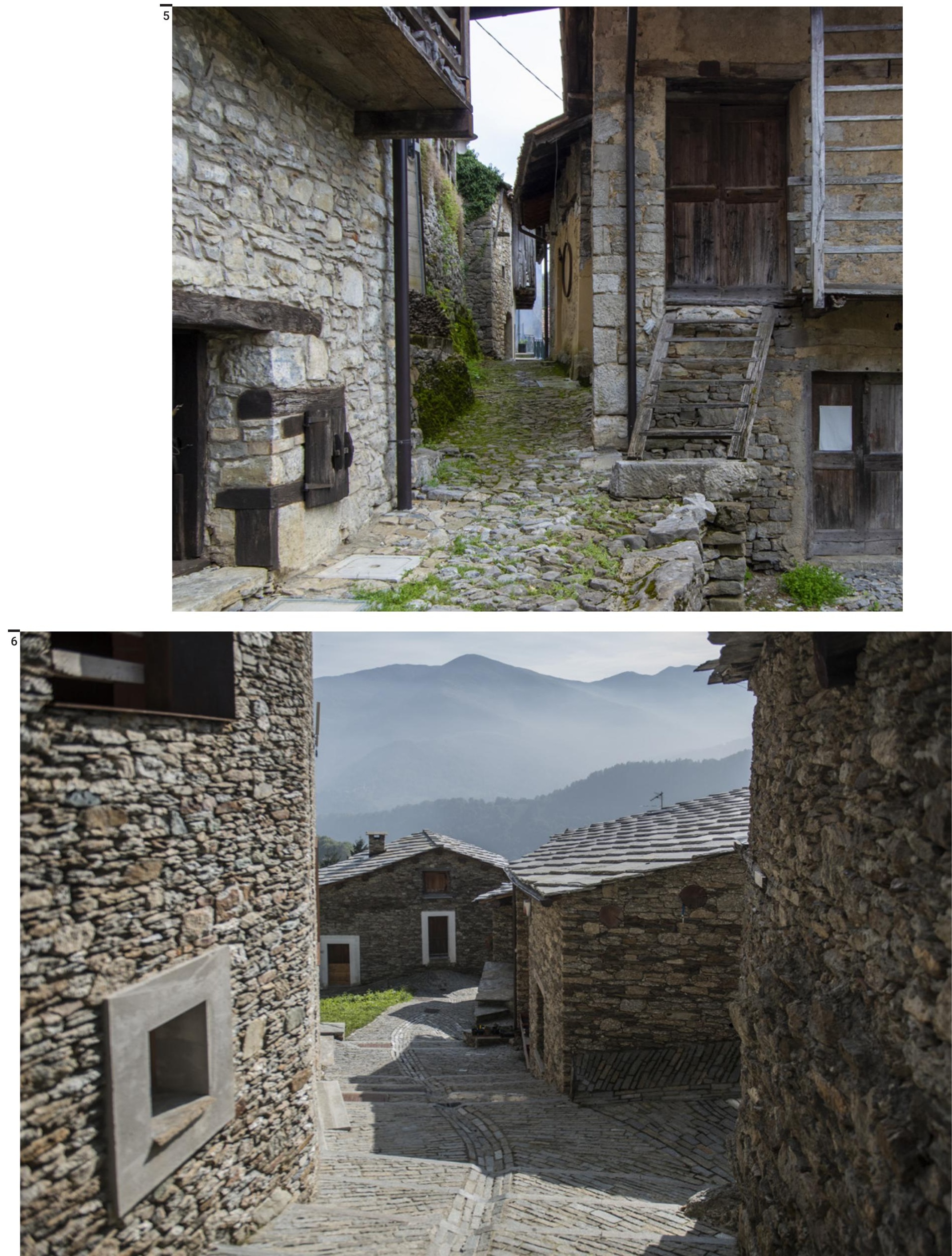